\title{
Interdisciplinary approach in management of edentulousness in menopausal women-a narrative review
}

\begin{abstract}
Menopause is a phase in women's life in which many changes in her body and surroundings occur simultaneously thereby causing an impact on her quality of life. Edentulousness adds to the vulnerability of such a patient. Apart from speciality skills a doctor/dentist should be equipped with appropriate communication skills for holistic management of the patient. A patient centered interdisciplinary approach with team work of gynaecologist, prosthodontist/ dentist, orthopaedician, communication skill expert, psychologist, counsellor and nutritional expert in managing edentulousness will help in achieving positive outcomes and improving the overall quality of life of the patient.
\end{abstract}

Keywords: Menopausal women, Edentulousness, Communication skill, Dental, Complete dentures, Psychological factors, Gynaecologist, Prosthodontist
Volume 5 Issue 6 - 2016

\author{
Varsha Murthy,' Sethuraman $K R,{ }^{2}$ Sunayana \\ Choudhury, ${ }^{3}$ Shakila $\mathrm{R}^{4}$ \\ 'Department of Prosthodontics and Implantology, Mahatma \\ Gandhi Medical College Campus, India \\ ${ }^{2}$ General Medicine, Sri Balaji Vidyapeeth, India \\ ${ }^{3}$ Department of Psychiatry, Mahatma Gandhi Medical College \\ and Research institute, India \\ ${ }^{4}$ Department of Prosthodontics and Crown \& Bridge, Mahatma \\ Gandhi Postgraduate Institute of Dental Sciences, India
}

\begin{abstract}
Correspondence: Varsha Murthy, Professor, Department of Prosthodontics and Implantology, Indira Gandhi Institute of Dental Sciences, Mahatma Gandhi Medical College Campus, Sri Balaji Vidyapeeth, Pondy-Cuddalore Main Road, Pillayarkuppam, Puducherry-607402, India, Tel $91-7708364959$ Emaildrvarshamurthy@gmail.com
\end{abstract}

Received: October 30, 2016 | Published: December 21, 2016
Abbreviations: BMS, Burning Mouth Syndrome; WHO, World Health Organization

\section{Introduction}

Complete edentulousness is a condition wherein a patient has complete loss of teeth in maxillary as well as mandibular arch. Prosthodontics is a branch of dentistry concerned with replacement or rehabilitation of such a condition with complete dentures. Success of complete denture treatment is multifactorial ${ }^{1}$ as it should meet patient's expectations and improve their quality of life. Diagnosis and treatment planning relies on thorough assessment of the patient's oral, physical and psychological condition and also technical and communication skills of the dentist. Complexity increases in presence of factors that enhance the problems associated with edentulousness. One such problem which occurs concurrently in women's life is menopause. The rate of bone loss in postmenopausal women predicts tooth loss i.e. for every $1 \%$ per year decrease in whole body bone mineral density, the risk of loss of teeth increases more than four times. ${ }^{2}$ Considering the age of menopausal women and expected complete tooth loss it is very likely that a dentist specially a prosthodontist (dentist specialised in treating complete denture patients) may encounter such patients frequently. ${ }^{3}$

The World Health Organization (WHO), has defined three age stages during the midlife age for women as Menopause, Premenopause and Perimenopause. ${ }^{4}$ Complete denture treatment in an women in any of these 3 stages or a patient for whom menopause has occurred prematurely should always incorporate a multidimensional approach and should not ignore oral, social and psychological changes that may also occur in a woman's life at the same time. It is important that dentists are aware of signs and symptoms of menopause and its treatment apart from the appropriate communication skills to be applied. The dentist should encourage patient centred practice by keeping in mind the patient as a whole and should refer all complete denture patients nearing or in menopausal or of post menopausal age for gynaecological consultations to improve their overall quality of life. Some of the major factors influencing the complete denture treatment, that a dentist should be aware of are:

\section{Oral factors}

The principal peri and postmenopausal oral symptoms are xerostomia, neuralgia, systemic diseases or medications causing fragile tissues, burning mouth syndrome (BMS), other mucosal disorders. neurological disorders. osteoporosis etc. ${ }^{5,6}$

Xerostomia or dry mouth may cause difficulty wearing removable dental prostheses ${ }^{7-10}$ and may need modifications in materials used for impression making and fabrication of denture.

Mucosal disorders: Due to decrease in estrogen levels the oral epithelial maturation process is affected during menopause, leading to thin and atrophic epithelium. ${ }^{11}$ In patients with xerostomia and sensitive mucosal tissues, BMS occurs as common dysesthesia (distortion of a sense), usual complaints by the patient are burning sensation in the oral cavity, in the absence of clinically apparent changes in the oral mucosa. The oral mucosal changes noticed may thus range from a condition referred to as "menopausal gingivostomatitis" to an atrophic pale appearing mucosa. ${ }^{8,12}$ It is characterized by gingiva that bleeds readily, with an abnormally pale dry/shiny erythematous appearance. Other oral mucosal disorders include candidiasis, pemphigus vulgaris, benign mucosal pemphigoid, lichen planus, and oral ulcerations following mechanical trauma due to abnormal oral habits and chronic denture-induced irritation., ${ }^{8,12}$ These mucosal disorders cause difficulty in impression making and denture wearing. 
Neurological disorders such as trigeminal neuralgia, atypical facial pain, alzeimers influence impression making procedures, jaw relation records, and denture retention. ${ }^{3}$

Osteoporosis: It has been found that women with severe osteoporosis are three times more likely to be edentulous than healthy, age-matched controls. ${ }^{15}$ Excessive bone loss due to osteoporosis may cause denture making and retention compromised.

Aging: General health also tends to deteriorate due to problems in mastication and avoidance of certain food due to the above oral factors, it gets aggravated more if in combination with loss of teeth. Aging process or edentulousness for a long time may cause irregular bone loss or resorption, tongue enlargement and changes in soft tissues such as the physical appearance of wrinkles, loss of vertical dimension and may contribute to a woman's negative body image. ${ }^{16,17}$ It may also give 'witches appearance' with mandible ahead than maxilla.

\section{Socio-cultural factors}

Social factors: A women's relationship with her partner, family, friends may also influence the experience of menopause. ${ }^{18}$ Widowed, separated and divorced women had higher rates of depression than married women. Women may have 'empty nest syndrome' because of children leaving home and for some who do not have children may get depressed because of lost hopes of childbearing beyond menopause. Depressed women around menopause period may have strained relationships, fewer friends and social support. Edentulousness is seen as a stigma and symbol of unesthetic appearance in many societies. Repeated enquiry about edentulousness by family members and relatives may cause embarrassment and self consciousness leading to covering of mouth while speaking or smiling, hesitant presentation in a social sphere by husband or children, speech difficulty, avoidance of going out or eating in a social function are the kind of social pressures a women may face.

Cultural factors: The influence of culture on menopause has been found by many researchers to be extensive. ${ }^{19}$ In developing countries, women in some cultures may take on more esteemed roles postmenopause, ${ }^{19}$ unlike other cultures where women's contribution to society may be devalued post menopause. ${ }^{20}$ Mainly in underdeveloped and developing countries women are suppressed and their needs are not given priority, with common statements from husbands or family members as "'Why do you need to replace teeth at this age, what more is left". Women are not allowed to be decision makers for themselves and are questioned and suspected if they go for dental treatment like complete dentures for frequent or multiple appointments.

Education and employment: Studies have shown that employed women reported less discomfort than unemployed women during the menopause. Better objective health outcome has been reported in women who felt that their work role was important than those who did not. ${ }^{21,22}$ Housewives have been found to have worse scores on psychosocial measures of quality of life compared to working women. ${ }^{23}$ Social class to which a woman belongs also have been found to play a role. However, unemployment and education have shown mixed result in their relationship with menopause. ${ }^{24-26}$

Family responsibilities: Sometimes due to other priorities in life, women tend to ignore their health, learn to adjust with the changes and consider it as routine and do not approach any doctor for discussing their problems with menopause and also postpone or deter to get the teeth replaced.

Financial support: Sometimes cost of treatment is not affordable by the patient due to own circumstances or being dependent on husband or children.

Support system: If patient's age is more, or the distance from the health services is more, it is very likely that somebody has to get them for treatment and the decision lies on the support system. Support of family plays a role in complete denture treatment.

\section{Psychological factors}

Depression, stress and anxiety: Many women present at the menopause with depression and stress, ${ }^{27}$ irritability and anxiety, increased sleep disturbance, thus affecting negative mood and anxiety ${ }^{28}$ along with mood swings, tiredness, irritability, and insomnia. ${ }^{29-31}$ Some researchers have reported that psychological symptoms, such as depressed mood, are experienced more severely at the perimenopause rather than post-menopause. ${ }^{32}$

Coping skills: Menopausal women have been found to not cope as well as non menopausal women with stress and reported increased negative mood as a consequence. . $^{33,34}$

Personality of the patient: It has been found that personality type was a predictor of psychological symptoms in a small sample of patients from a menopause clinic. ${ }^{33}$ Patients personality plays a major role in satisfaction with complete dentures. ${ }^{1}$

\section{Other factors}

Expectations: Patients unrealistic expectations of artificial dentures to look and function as original teeth because of inadequate information.

Previous experiences: Previous denture and dentist experiences also influence the treatment and acceptance of new complete dentures. ${ }^{1}$ However all the above factors may be missing or overlapping in a particular individual and therefore combination approach has to be practised.

\section{Discussion}

With all the above multiple factors playing a role while treating a menopausal complete denture patients, it becomes necessary that the dentist is equipped with appropriate communication skills apart from speciality/technical skills required for making a complete denture. It was found in one of the study that postmenopausal women were not aware of their periodontal health and the risks involved to their systemic health and therefore authors suggested creating a taskforce comprising of periodontists, generalists, communication experts, and women at large, to develop informational guidelines. ${ }^{35}$

Dentists need to treat such patients with care taking into account the systemic changes, which the patient is undergoing. Women need to be educated regarding the changes in the oral cavity and body and the care to be taken. To make all this possible dentists and gynaecologist need to work in close consultation with each other by referring postmenopausal women with oral symptom to a dentist and to a gynaecologist for medical intervention if necessary. ${ }^{36}$ Therefore, management of such patients requires special care and attention.

\section{Dental management of menopause}

i. In case of xerostomia, patient should be encouraged to have abundant water intake, sugar-free sweets, chewing gum and sialogogues (pilocarpine, bromhexine or bethanechol) to increase salivation. ${ }^{3,5,14,37}$ Adequate oral hygiene maintenance should be taught. 
ii. In cases of burning mouth due to acrylic denture base allergy, it may be replaced with a different material e.g. metal denture base. $^{38}$

iii. In case of atrophic or sensitive mucosa, denture should be fabricated as smooth as possible to avoid traumatizing the fragile mucosa. ${ }^{39}$

iv. The symptoms of mucosal disorders necessitate a scrupulous assessment of denture fit and evaluation of the status of underlying tissues to eliminate chronic irritation. ${ }^{13}$ Reservoir denture or flexible dentures can be suggested as option. ${ }^{40,41}$

v. In denture wearing patients, a careful evaluation of denture fit and the foundation tissues is indicated, since denture adjustment or replacement may help to eliminate chronic irritation. ${ }^{6}$

vi. Tooth loss in menopausal and postmenopausal women might signal the onset of systemic osteoporosis and should be referred to the gynaecologist and orthopedician for the necessary treatment. Dentists should refer postmenopausal women with eroded cortex or thin cortical width $(<3.0 \mathrm{~mm})$ for bone densitometry. ${ }^{42}$

vii. Edentulous women visiting dentist frequently with particular problems of ill-fitting dentures ( due to alveolar ridge regression) should not be categorised as having personality disorder. Careful assessment and examination should be done to rule out osteoporotic changes. Osteoporotic patients require new dentures much more often than non osteoporotic patients. ${ }^{43}$

\section{Therapeutic management of menopause}

A therapeutic relationship should be established with the woman undergoing menopausal changes in order to help to make her treatment and the experience of menopause more positive. Each woman will have her own oral conditions and limitations, personal and psychological history, life events, coping skills, family background, relationship history, body image, roles in family and working place, social and cultural interpretation of how menopause affects her life and therefore dentists should assess the information regarding the same. Studies have shown that that patients want further education and information, support, validation and for someone to take the time to listen to their individual experience. ${ }^{44}$ Some of them even need further counselling along with referral to a qualified psychologist in combination with the treatment provided by a physician. ${ }^{18} \mathrm{An}$ interdisciplinary approach by the dentist, gynaecologist, nutrition counsellor and psychologist should focus on following approaches:

a. Gynaecologist should encourage their patients to seek regular dental evaluation for early diagnosis, prevention and management of oral disorders due to menopause.

b. Dentist should be aware of potential effects of menopause and its treatments on bone and dental health. ${ }^{45}$ and should refer their patients to gynaecologist for needful regarding their systemic health.

c. In case if dentist perceives mood disorders and signs of depression, anxiety and stress, multiple dentures (rule out any problem with the denture), while doing treatment or interacting with they can be referred for psychological counselling.

d. Changes in dietary habits may be due to effects of menopause or due to avoidance of certain food because of edentulousness or limitations due to denture wearing. Therefore a careful assessment of diet of patient and nutritional dietary counselling is necessary. e. In case of patients with neurological disorders they should be explained about the procedures (impression making, jaw relation) and counselled for fear, anxiety and stress reduction.

f. Dentist should develop listening skills to allow patient to tell about story and circumstances. This may reveal some important information which may not be given by patient as such.

g. Many menopausal women present to doctor with many symptoms such as depression and anxiety believing that they are caused by menopause. They may not do the same when they visit a dentist. Careful questioning skills as non leading, indirect and probing questions may help in finding out if patients is undergoing or had any depression, anxiety or stress in the recent past.

h. Any previous experience of mood disorders, depression, anxiety, stress, a negative attitude to menopause and ageing, negative life events should be questioned

i. Questioning and listening skills are also required to elicit patients coping ability. It can be found out by asking patient as to how they coped up with problems in her life. This information will give an idea to the dentist about how well the patient will be able to cope with the changes due to the new denture.

j. Personality type plays a major role in complete denture treatment and its identification early helps in avoiding dissatisfaction with the denture as well as the dentist.

k. Womens view about her body image should be enquired to know how much rehabilitating with denture may be helpful to patients and if women experience negative body image, low self esteem and depression they can be referred to a psychologist or counsellor

1. Questioning skills should be used to find out about their relationship with their family make-up and background (partner, children, friends, elders, in laws etc), and how they feel about their relationship with them.

m. Ask women about the roles they have in their life, if they are satisfied with these roles, and their interest as this may help in rapport building as well as bring positive change in their lives.

n. Assessment of the social background and culture of the menopausal patient may help the dentist to find out about patient limitations and difficulties for treatment visits. In such cases flexible number of appointments (e.g. multiple steps in a single day) and faster appointments can be planned.

o. Good rapport building and empathetic approach with each patient will help in achieving a greater patient satisfaction with the dentist as well as the denture as each individual has a new story and background.

p. Patient's primary agenda and fear any for seeking the treatment should be elicited to obtain better cooperation and satisfying them.

q. Patient's non verbal expressions and body language should be noticed to find out any discomfort, or disinterest.

r. Elicit expectations of the patient from new as well as previous dentures and dentists.

s. Emphatetise and understand limitations of patient to perform jaw movements during various procedures like impression making and jaw relation. 
t. Assess oral health related quality of life of the patient before and after the treatment.

u. Allow patient to participate in decision making of treatment plan and during procedures (e.g. final try in) specially decision about their future esthetics for a positive outcome.

v. Educate patient and their family members regarding the effects of menopause through educational material which is self explanatory using pictures, videos or role play etc

w. Explain them about the benefits of regular follow up appointments after giving dentures and motivate them to follow the instructions given.

$\mathrm{x}$. Finally an interdisciplinary approach is required wherein the gynaecologist should notice patients partially dentulous or edentulous state and give referral to the dentist. Similarly dentist should be aware of associated problems with menopause and refer to the gynaecologist for appropriate treatment.

\section{Conclusion}

The need for an interdisciplinary approach (prosthodontist, medicine expert, gynaecologist, psychologist, nutrition counsellor and communication skill expert) while dealing with an pre, peri or menopausal women cannot be undermined. Sometimes dentist may have to play a major role since such a patient would have reported to $\mathrm{him} /$ her first for replacement of teeth. Such patients may be unaware of oral, systemic and psychological changes or manifestations of menopause. Therefore dentist-patient communication should appropriately incorporate skills like eliciting agenda or fear, questioning, listening, answering, motivating, counselling, empathetising, persuasion, picking up non verbal cues, understanding body language etc. Therefore it requires a multidimensional approach to treat the menopausal woman.

\section{Acknowledgments}

None.

\section{Conflicts of interest}

None.

\section{References}

1. Marchini L. Patients' satisfaction with complete dentures: an update. Braz Dent Sci. 2014;17(4):5-16.

2. Krall EA, Garcia RI, Dawson-Hughes B. Increased risk of tooth loss is related to bone loss at the whole body, hip and spine. Calcif Tissue Int 1996;59(6):433-437.

3. Mutneja P, Dhawan P, Raina A, et al. Menopause and the oral cavity Indian J Endocrinol Metab. 2012;16(4):548-551.

4. World Health Organ Tech Rep Ser. Research on the Menopause in the 1990's. Report of a WHO Scientific Group. Geneva, Switzerland. 1996;p.1-79.

5. Meurman JH, Tarkkila L, Tiitinen A. The menopause and oral health Maturitas. 2009;63(1):56-62.

6. Frutos R, Rodríguez S, Miralles-Jorda L, et al. Oral manifestations and dental treatment in menopause. Med Oral. 2002;7(1):26-30,31-35.

7. Santosh P, Nidhi S, Sumita K, et al. Oral findings in postmenopausal women attending dental hospital in Western part of India. J Clin Exp Dent. 2013;5(1):e8-e12.
8. Friedlander AH. The physiology, medical management and oral implications of menopause. J Am Dent Assoc. 2002;133(1):73-81.

9. Laing LP, Koka S. Systemic health aspects and nutritional considerations for edentulous patients. In: Zarb G, et al. (Eds.), Prosthodontic Treatment for Edentulous Patients: Complete Dentures and Implant-Supported Prostheses. (13th edn), St. Louis, Mosby, Elsevier Inc. 2013;p.28-34.

10. Akintoye SO, Collins MT, Ship JA. Diabetes mellitus and Endocrine diseases. In: Greenberg MS, Glick M, Ship JA, editors. Burket's Oral Medicine. (11th edn). Hamilton: BC Decker Inc. 2008;pp.509-535.

11. Agha-Hosseini F, Mirzaii-Dizgah I, Mansourian A, et al. Relationship of stimulated saliva 17beta-estradiol and oral dryness feeling in menopause. Maturitas. 2009;62(2):197-199.

12. Paganini-Hill A. Hormone therapy and oral health. Menopause Manag. 2007;16:31-40.

13. Portillo GM. Oral manifestations and dental treatment in menopause. Med Oral. 2002;7:31-35.

14. Chaveli López B, Sarrión Pérez MG, Jiménez Soriano Y. Dental considerations in pregnancy and menopause. $J$ Clin Exp Dent. 2011;3(2):135-144.

15. Kribbs PJ. Comparison of mandibular bone in normal and osteoporotic women. J Prosthet Dent. 1990;63(2):218-222.

16. Janelli LM. Are there body image differences between older men and women? Western Journal of Nursing Research. 1993;15(3):327-339.

17. Bannister EM. Women's midlife confusion: 'why am I feeling this way?' Issues in Ment Health Nurs. 2000;21(8):745-764.

18. Deeks AA, McCabe MP. Menopausal stage and age and perceptions of body image. Psychol Health. 2001;16:367-379.

19. Beyene Y. Cultural significance and physiological manifestations of menopause. A biocultural analysis. Cult Med Psychiatry. 1986;10(1):4771.

20. Berkun CS. In behalf of women over 40: understanding the importance of the menopause. Social Work. 1986;31(5):378-384.

21. Krystal S, Chiriboga DA. The empty nest process in mid-life men and women. Maturitas. 1979;1(3):215-222.

22. Gannon L. Menopausal symptoms as consequences of dysrhythmias. Journal of Behavioral Medicine. 1993;16(4):387-402.

23. Blumel JE, Castelo-Branco C, Binfa L, et al. Quality of life after the menopause: a population study. Maturitas. 2000;34(1):17-23.

24. Hunter M, Battersby R, Whitehead M. Relationships between psychological symptoms, somatic complaints and menopausal status. Maturitas. 1986;8(3):217-228.

25. McKinlay JB, McKinlay SM, Brambilla D. The relative contributions of endocrine changes and social circumstances to depression in mid-aged women. Journal of Health and Social Behavior. 198728(4):345-363.

26. Dennerstein L. Psychosocial and mental health aspects of women's health. World Health Statistical Quarterly. 1993;46:234-236.

27. Liao KL, Wood N, Conway GS. Premature menopause and psychological well-being. J Psychosom Obstet Gynaecol. 2000;21(3):167-174.

28. Owens JF, Matthews KA. Sleep disturbance in health middle-aged women. Maturitas. 1998;30(1):41-50.

29. Oldenhave A, Jaszmann LJ, Haspels AA, et al. Impact of climacteric on well-being. A survey based on 5213 women 39 to 60 years old. Am J Obstet Gynecol. 1993;168(3 Pt 1):772-780.

30. Stearns V, Ullmer L, López JF, et al. Hot flushes. Lancet. 2002;360(9348):1851-1861. 
31. Deecher DC, Dorries K. Understanding the pathophysiology of vasomotor symptoms (hot flushes and night sweats) that occur in perimenopause, menopause, and postmenopause life stages. Arch Womens Ment Health. 2007;10(6):247-257.

32. Vanwsenbeeck I, Vennix P, van de Wiel H. Menopausal symptoms: association with menopausal status and psychosocial factors. $J$ Psychosom Obstet Gynaecol. 2001;22(3):149-158.

33. Collins A, Hanson U, Eneroth P. Postmenopausal symptoms and response to hormonal replacement therapy: influence of psychological factors. Journal of Psychosomatics and Obstetrics. 1983;2(4):227-233.

34. Ballinger SE. Psychosocial stress and symptoms of menopause: a comparative study of menopause clinic patients and non-patients. Maturitas. 1985;7(4):315-327.

35. Palomo L, Chitguppi R, Buencamino MC, et al. A need to educate postmenopausal women of their periodontal health. J Indian Soc . 2013;17(2):225-227.

36. Newadkar UR. Mouth on fire: Oral discomfort in postmenopausal women may be surprising!! J Midlife Health. 2015;6(4):184-186.

37. Dutt P, Chaudhary S, Kumar P. Oral health and menopause: A comprehensive review on current knowledge and associated dental management. Ann Med Health Sci Res. 2013;3(3):320-323.
38. Bhatia V, Bhatia G, Jain N, et al. An innovative metal base denture design for a 55-year-old menopausal woman. J Nat Sci Biol Med. 2013;4(2):468-472.

39. Chiramana S, Ashok K. Examination, diagnosis and treatment planning for complete denture therapy: A review. J Orofac Sci. 2010;2(3):29-35.

40. Mendoza AR, Tomlinson MJ. The split denture: a new technique for artificial saliva reservoirs in mandibular dentures. Aust Dent J. 2003;48(3):190-194.

41. Murthy V, Yuvraj V, Nair PP,et al. Prosthodontic management of radiation induced xerostomic patient using flexible dentures. BMJ Case Rep. 2012:pii:bcr1120115250.

42. Taguchi A, Tsuda M, Ohtsuka M, et al. Use of dental panoramic radiographs in identifying younger postmenopausal women with osteoporosis. Osteoporos Int. 2006;17(3):387-394.

43. Zachariasen RD. Oral manifestations of menopause. Compendium. $1993 ; 14(12): 1584$.

44. Walters CA. The psychosocial meaning of menopause: women's experiences. J Women Aging. 2000;12(3-4):117-131.

45. Buencamino MCA, Palomo L, Thacker HL. How menopause affects oral health, and what we can do about it. Cleve Clin J Med. 2009;76(8):467475 . 